\title{
The Effect of Calf Stiffness on Gait, Foot Pressure and Balance in Adults
}

\author{
JeonHyeong Lee ${ }^{1}$, JongSung Chang ${ }^{2}$ \\ 'Department of Physical Therapy, Daegu Health College, Daegu; ${ }^{2}$ Department of Physical Therapy, Yeungnam University College, Daegu, Korea
}

Purpose: The purpose of this study was to investigate the effects of calf tightness on gait, plantar pressure, and balance in adults.

Methods: A total of 60 participants were divided into a normal group of 30 subjects with normal dorsiflexion angle (20-25 degrees) and an experimental group of 30 subjects with limited dorsiflexion angle (0-15 degrees) due to calf tightness. Gait ability and foot pressure of the subjects was measured with a treadmill, and the balance ability was measured by PROKIN system.

Results: A significant difference in COP length, loading response, and single limb support was observed between groups. The COP length and single limb support ratio in the normal group was greater than in the experimental group, but the experimental group showed a higher ratio for loading response in the gait ratio.

Conclusion: Our results indicated that calf tightness was negative effects on balance and gait ability, so assessment of the muscle tightness should be considered during exercise and treatment.

Keywords: Balance, Calf stiffness, Gait, Treadmill

\section{INTRODUCTION}

At the time of sudden postural perturbation, normal adults are able to recover postural stability through appropriate postural strategies using appropriate agonists and antagonists. ${ }^{1,2}$ Postural stability refers to an ability to maintain and control the center of mass within the area in contact with the base of support. ${ }^{1-3}$ This postural stability is divided into static balance and dynamic balance, and for its normal control, efficient integration of sensory, motor, and central nerve systems is required. ${ }^{3}$ It is also affected by the environment, task demands, strength, and the range of motion. ${ }^{2}$

Muscle tightness is a typical component of the motor system that affects gait or balance, where the muscle's ability to contract and relax decreases, causing narrower ranges of motion for the acting joint than normal and the muscle length is shortened, limiting movement in the direction of extension. ${ }^{3,4}$ In addition, muscle tightness primarily occurs in people who failed to receive adequate rehabilitation from sustained muscle injuries or who lack physical activity.

Received Nov 4, 2019 Revised Dec 4, 2019

Accepted Dec 5, 2019

Corresponding author JongSung Chang

E-mail changjs@ync.ac.kr
Muscle tightness makes muscles and tendons more susceptible to injuries and limits the movement of the joint where the muscle is involved, causing functional problems. Muscle tightness in the legs, in particular, is frequently observed in the hamstring and the calf consisting of muscles that pass more than two joints, as a result, it negatively affects gait and balance and is also accompanied by proprioceptive sensory problems. ${ }^{3,4}$

Reduced range of motion of the ankle for dorsiflexion due to calf tightness leads to difficulties in maintaining the center of mass in the weight-bearing posture, and such limited dorsiflexion induces a compensatory movement in the ankle, resulting in leg deformation. ${ }^{3-5}$ In addition, calf tightness can make it difficult for the heel to push the ground properly during walking, running, jumping, or climbing stairs. Problems in performing an ankle strategy may also occur when small body disturbance occurs. ${ }^{3}$

Recently, many studies reported that reduced ankle range of motion affects physical activities and walking, and various clinical features such as increased risk of falls, ${ }^{6}$ decreased walking speeds, ${ }^{7}$ and

Copylight (C)2019 The Korean Society of Physical Therapy

This is an Open Access article distribute under the terms of the Creative Commons Attribution Non-commercial License (Http:// creativecommons.org/license/by-nc/4.o.) which permits unrestricted non-commercial use, distribution, and reproduction in any medium, provided the original work is properly cited. 
heel rise in the early stance phase. ${ }^{8}$ In addition, various stretching exercises were proposed as an intervention for reduced ankle range of motion. Although there are various studies on the therapeutic intervention and clinical conditions of calf muscle shortening, ${ }^{9-12}$ studies on detailed clinical aspects such as gait stage, movements of foot pressure, and anterior-posterior and left-right balance are insufficient. And the detailed results of clinical features are effective in selecting more efficient and effective interventions. Therefore, this study aims to investigate the effects of calf tightness on gait, plantar pressure, and balance in adults.

\section{METHODS}

\section{Subjects}

The subjects of this study were selected from students attending D University located in D city and those with no specific disease in the musculoskeletal and nervous systems and those with no pain in the leg were selected. A total of 60 participants selected were divided into a normal group of 30 subjects with angles of joint motion that did not limit dorsiflexion (20-25 degrees) and an experimental group of 30 subjects with angles of joint motion that limited dorsiflexion (0-15 degrees) due to calf tightness, and then they underwent the experiment. Because Elveru et al. ${ }^{13}$ defined calf muscle shortening as passive ankle range of motion less than 10 degrees, and Novacheck ${ }^{14}$ suggested that the range of motion of dorsiflexion for normal gait should be maintained at about 20 degrees, we classified the calf muscle tightness from 0 to 15 degree. They were given a sufficient explanation on the purposes of this study and gave informed consent prior to investigations in this study.

\section{Experimental methods}

\section{(1) Range of motion}

A goniometer that measures the angle of movement of each joint in the human body was used to determine whether the subject's dorsiflexion was limited or not. The subject was seated on the bed with their leg being extended without touching the ground and was instructed to relax their ankle joint and to maintain a 90 degrees neutral position. Next, while the axis was placed on the outer malleolus and the stationary arm was placed on the fibular, the moving arm was placed in the side line of the sole of the foot relative to the fifth toe. ${ }^{15}$ In this state, the subject performed dorsiflexion, during which the corresponding values were measured. Then, the data obtained were sorted by normal and experimental groups.

\section{(2) gait capacity}

Zebris FDM-T treadmill (Zebris Medical GmbH, Germany) (Figure 1) was used to measure the gait capacity of the subject. This machine was equipped with a mat installed with a pressure sensor, which was attached underneath the treadmill belt and could control speed from 0.2 to $22 \mathrm{~km} / \mathrm{hr}^{16}$ The input signal was converted to spatiotemporal values during walking and then was displayed as a graph, high reliability has been shown at double stance (0.85), step width (0.96), stride length (0.93), gait velocity (0.87), and stride time (0.87). ${ }^{17}$

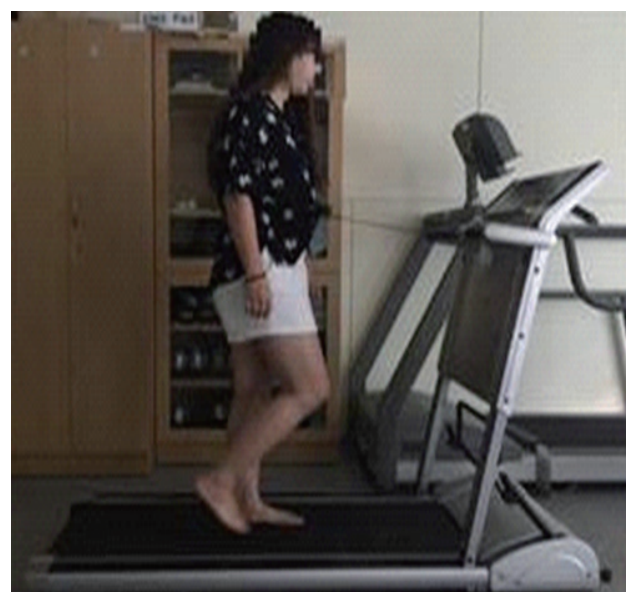

Figure 1. Zebris FDM-T treadmill.

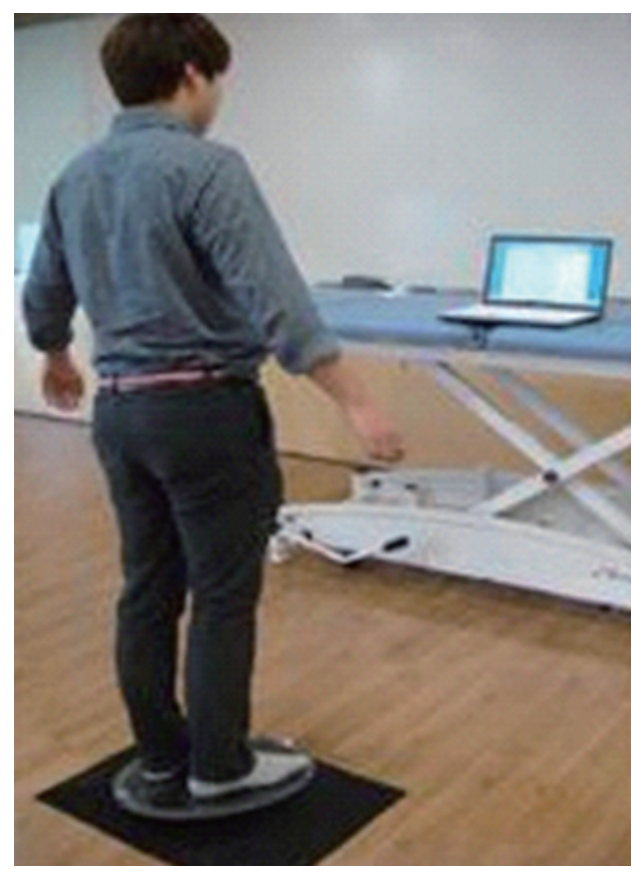

Figure 2. FROKIN System. 
Before the measurement, the subject was instructed to determine the speed at which they could walk most comfortably on the treadmill. After warming up for about 1 minute, the subject walked on the treadmill for 30 seconds with their eyes looking straight ahead, during which the measurement was taken. While the measurement was taken, the subject wore a safety clip device that can automatically stop the machine when they fall around their waist for the safety of the subject. To evaluate the gait capacity data, stride length, step width, COP length, stride time, cadence, and gait rate were analyzed and compared between groups.

\section{(3) balance capacity}

The PROKIN System (Prokin PK 200 WL, Italy) (Figure 2) was used to measure the subject's dynamic balance capacity. This instrument provides an assessment of the proprioceptive sensation associated with the balance capacity through the sensors installed on the foot plate while the subject stands on it. ${ }^{18}$

The subject was instructed to place their feet on the measuring instrument equipped with sensors in a standing position, to look straight ahead at the target line, and to maintain the posture not to fall for 10 seconds. The balance measurement was taken three times for 10 seconds each time and the average value was used for analysis. To evaluate the data obtained from the measurement, the anterior- posterior and medio-lateral movement angles, motion speed, and perimeter length were compared between groups.

\section{Statistics}

The collected data was means and standard deviations by descriptive statistics using SPSS 18.0 (SPSS Inc., Chicago) for windows. An independent t-test was performed to compare the changes in gait, plantar pressure, and balance capacity values between groups after confirming normality with the Shapiro-Wilks test. The statistical significance was accepted for p-values of less than 0.05 .

\section{RESULTS}

\section{General characteristics}

A total of 60 subjects were enrolled either an experimental group (15 men and 15 women) or a control group (15 men and 15 women). Table 1 display the gender, age, height, and weight of the groups. The results of the group analysis show that there were no significantly differences between two groups in height and weight ( $\mathrm{p}>$ $0.05)$.

\section{Comparison of gait capacity}

A significant difference in COP length was observed between

Table 1. General characteristics of subjects

\begin{tabular}{lccc}
\hline & Normal group & Experimental group & $\mathrm{t}$ \\
\hline Gender (Male/Female) & $15 / 15$ & $15 / 15$ & 2.21 \\
Age (year) & $22.77 \pm 2.56$ & $21.50 \pm 1.81$ & $0.01^{*}$ \\
Height $(\mathrm{cm})$ & $167.6 \pm 7.43$ & $166.3 \pm 8.30$ & 0.64 \\
Weight $(\mathrm{kg})$ & $60.20 \pm 10.77$ & $62.03 \pm 0.82$ & 0.66 \\
\hline
\end{tabular}

Mean $\pm S D,{ }^{*} p<0.05$

Table 2. Comparison of gait capacity

\begin{tabular}{|c|c|c|c|c|}
\hline & Normal group & Experimental group & $\mathrm{t}$ & $\mathrm{p}$ \\
\hline Stride length (cm) & $94.26 \pm 14.49$ & $93.83 \pm 8.48$ & 0.14 & 0.44 \\
\hline Step width (cm) & $10.15 \pm 2.04$ & $10.62 \pm 3.48$ & 0.68 & 0.24 \\
\hline COP length (mm) & $118.0 \pm 11.98$ & $112.6 \pm 11.62$ & 1.75 & $0.04^{*}$ \\
\hline Stride time (sec) & $1.17 \pm 0.09$ & $1.14 \pm 0.11$ & 1.23 & 0.11 \\
\hline Cadence (step/min) & $51.47 \pm 3.98$ & $53.30 \pm 5.10$ & 1.32 & 0.09 \\
\hline Stance phase (\%) & $63.80 \pm 1.61$ & $64.03 \pm 1.43$ & 0.59 & 0.27 \\
\hline Loading response & $13.33 \pm 1.22$ & $13.95 \pm 1.30$ & 1.92 & $0.02^{*}$ \\
\hline Single limb support & $36.89 \pm 1.37$ & $36.20 \pm 1.38$ & 1.94 & $0.02^{*}$ \\
\hline Pre-swing & $13.58 \pm 1.60$ & $13.90 \pm 1.33$ & 0.84 & 0.20 \\
\hline Swing phase(\%) & $36.20 \pm 1.61$ & $35.97 \pm 1.43$ & 0.59 & 0.27 \\
\hline
\end{tabular}

COP: center of pressure. Mean \pm SD, ${ }^{*} p<0.05$. 
Table 3. Comparison of balance capacity

\begin{tabular}{lcccc}
\hline & Normal group & Experimental group & $\mathrm{t}$ & $\mathrm{p}$ \\
\hline Anterior-posterior equilibrium $\left(^{\circ}\right)$ & $1.01 \pm 2.23$ & $0.41 \pm 2.27$ & 1.03 & 0.15 \\
Medio-lateral equilibrium $\left(^{\circ}\right)$ & $0.73 \pm 2.19$ & $1.02 \pm 1.98$ & 0.53 & 0.29 \\
Motion speed $(\mathrm{cm} / \mathrm{s})$ & $11.03 \pm 2.75$ & $11.60 \pm 3.93$ & 0.65 & 0.25 \\
Perimeter length $(\mathrm{mm})$ & $110.33 \pm 27.54$ & $116.01 \pm 39.32$ & 0.65 & 0.25
\end{tabular}

Mean $\pm S D,{ }^{*} p<0.05$

groups $(\mathrm{p}<0.05)$ (Table 2$)$, the COP length in the normal group was longer than in the experimental group. But no significant difference were found for stride length, step width, stride time, and cadence $(\mathrm{p}>0.05)$.

Comparison of the gait rate between groups, a significant difference in loading response and single limb support was observed $(\mathrm{p}<0.05)$, but no significant difference was found for pre-swing and swing phase $(\mathrm{p}>0.05)$ (Table 2). The normal group showed a higher single limb support ratio, but the experimental group showed a higher ratio for loading response in the gait ratio.

\section{Comparison of balance capacity}

There are no statistically significant differences in the anterior-posterior and medio-lateral movement angles, motion speed, and perimeter length between groups $(\mathrm{p}>0.05)$ (Table 3$)$.

\section{DISCUSSION}

The dorsiflexion of ankle joints and calf flexibility are essential elements for daily activities, such as gait and sports activities, it has been shown that muscle tightness of the calf leads to a decrease in the range of motion for dorsiflexion, and the resulting pain and decreased performance can cause changes in the gait pattern. ${ }^{19,20}$ In this study, the effects of limited dorsiflexion due to calf tightness on gait capacity, plantar pressure, and balance capacity wer e compared and analyzed. The results revealed that in the category of gait capacity, the center of pressure (COP) length and the single limb support ratio were higher in the normal group, while the loading response was higher in the experimental group. However, no differences between groups were found in balance capacity.

In order to analyze the plantar pressure and the gait, pressure movement and general characteristics of gait were measured. Plantar pressure measurement is widely used because it can measure the distribution of pressure applied to the foot and the trajectory path of the center of the body by using the pressure on the foot and gait evaluation can be performed on the treadmill without locational constraints. ${ }^{16}$ This study showed that the COP length was longer in the normal group than in the experimental group. It can be explained by the fact that during the anterior-posterior movement of ankle joints, weight was fully shifted from dorsiflexion to plantar flexion in the normal group, while forward weight shift was restricted by the limited range of motion in the experimental group. In the gait ratio, the normal group showed a higher single limb support ratio, while the experimental group showed a higher ratio for loading response. This indicates that the experimental group had a longer time for weight shift and double support, which was caused by the decreased calf muscle's ability to control eccentric contraction and its reduced flexibility due to muscle tightness. Jung ${ }^{21}$ reported that the normal range of motion for ankle is $50^{\circ}$ for plantar flexion and $20^{\circ}$ for dorsiflexion and proper gait requires plantar flexion of at least $20^{\circ}$ and dorsiflexion of at least $10^{\circ}$.

Although no significant inter-group difference was found in balance capacity of the two groups, the experimental group showed greater medio-lateral equilibrium, motion speed, and perimeter length, while the normal group showed greater anterior-posterior equilibrium. Reduced ankle mobility with calf muscle tightness in experimental group was maintained in the medio-lateral equilibrium than anterior-posterior equilibrium for static balance, thus contributing to the better anterior-posterior balance observed in the experimental group. In the experimental group, only modest decreases in other balance capacity items were observed, except for the anterior-posterior item, which is believed to be due to the fact that although calf tightness lowered balance, the subjects were young and had no severe limitations, such as ankle contracture. Nevertheless, improvements in the range of motion and balance capacity have been reported after the application of treatments, such as calf massage, stretching, and ankle joint mobilization, ${ }^{21-24}$ so calf tightness is considered to have a negative effect on balance and requires 
intervention.

Calf tightness was negative effects on balance and gait ability, so assessment of the muscle tightness should be considered during exercise and treatment. In the future further studies are needed to explore the relationship between calf tightness and balance or gait ability in a wide spectrum of subjects with different ages.

\section{REFERENCES}

1. Jacobs JV, Horak FB. External postural perturbations induce multiple anticipatory postural adjustments when subjects cannot pre-select their stepping foot. Exp Brain Res. 2007;179(1):29-42.

2. Jeon HS, Hwang S, Woo YK. The effect of ankle and knee immobilization on postural control during standing. Knee. 2013;20(6):600-4.

3. Shah C. The effect of hamstring and calf tightness on static, dynamic balance and mobility - a correlation study. Indian J Pysiother \& Occup Ther. 2013;7(4):17-22.

4. Murphy DF, Connolly DA, Beynnon BD. Risk factors for lower extremity injury: A review of the literature. Br J Sports Med. 2003;37(1):13-29.

5. Lima YL, Ferreira V, de Paula Lima PO et al. The association of ankle dorsiflexion and dynamic knee valgus: a systematic review and metaanalysis. Phys Ther Sport. 2018;29:61-9.

6. Johnson E, Bradley B, Witkowski K et al. Effect of a static calf muscletendon unit stretching program on ankle dorsiflexion range of motion of older women. J Geriatr Phys Ther. 2007;30(2):49-52.

7. Hunter GR, McCarthy JP, Bamman MM. Effects of resistance training on older adults. Sports Med. 2004;34(5):329-48.

8. Karas M, Hoy D. Compensatory midfoot dorsiflexion in the individual with heelcord tightness: Implications for orthotic device designs. J Prosthet Orthot. 2002;14(2):82-93.

9. Gajdosik RL. Relation of age and passive properties of an ankle dorsiflexion stretch to the timed one-leg stance test in older women. Percept Mot Skills. 2006;103(1):177-82.

10. Power K, Behm D, Cahill F et al. An acute bout of static stretching: Effects on force and jumping performance. Med Sci Sports Exerc. 2004; 36(8):1389-96.
11. Samukawa M, Hattori M, Sugama N et al. The effects of dynamic stretching on plantar flexor muscle-tendon tissue properties. Man Ther. 2011;16(6):618-22.

12. Thacker SB, Gilchrist J, Stroup DF et al. The impact of stretching on sports injury risk: A systematic review of the literature. Med Sci Sports Exerc. 2004;36(3):371-8.

13. Elveru RA, Rothstein JM, Lamb RL. Goniometric reliability in a clinical setting. Subtalar and ankle joint measurements. Phys Ther. 1988;68(5): 672-7.

14. Novacheck TF. The biomechanics of running. Gait Posture. 1998;7(1): 77-95.

15. Martin RL, McPoil TG. Reliability of ankle goniometric measurements: A literature review. J Am Podiatr Med Assoc. 2005;95(6):564-72.

16. Kim SG. The effect of action observation exercise and cognitive therapy exercise on balance and gait in stroke patients. Daegu University, Dissertation of Doctorate Degree. 2014.

17. Faude O, Donath L, Roth R et al. Reliability of gait parameters during treadmill walking in community-dwelling healthy seniors. Gait Posture. 2012;36(3):444-8.

18. Amico AP, Nisi M, Covelli I et al. Efficacy of proprioceptive training with prokin system in balance disorders from multiple sclerosis. J Mult Scler. 2014;1(2):1-10.

19. Jeon IC, Kwon OY, Yi CH et al. Ankle-dorsiflexion range of motion after ankle self-stretching using a strap. J Athl Train. 2015;50(12):1226-32.

20. Kuzma SA, McNeil SP. A comparison of prostretch versus incline board stretching on active ankle dorsiflexion range of motion. UW-L Journal of Undergraduate Research VIII. 2005;1:1-6.

21. Jeong HC, Lim NY. Effect of taping therapy on the rom, pain, and discomfort of adults with ankle pain. J Muscle Joint Health. 2010;17(2): 124-31.

22. Roh HL. Effects of calf muscle massage on the balance. J Kor Clini Health Scie. 2015;3(4):490-4.

23. Smajla D, Garcia-Ramos A, Tomazin K et al. Selective effect of static stretching, concentric contractions, and a balance task on ankle force sense. PLoS One. 2019;14(1):e0210881.

24. Divya J, Kiruthika, Kumaresan. Influence of ankle exercise on balance among community dwelling older adults. Res J Pharm \& Tech. 2018; 11(7):2935-9. 